\title{
ChemComm
}

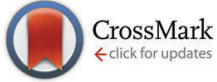

Cite this: Chem. Commun., 2015, 51,13193

Received 5th June 2015,

Accepted 9th July 2015

DOI: $10.1039 / \mathrm{c} 5 \mathrm{cc} 04653$

www.rsc.org/chemcomm

\section{Brilliant glyconanocapsules for trapping of bacteria $\dagger$}

\author{
Xibo Yan, ${ }^{a b c}$ Adeline Sivignon, ${ }^{\text {de }}$ Pierre Alcouffe, ${ }^{\text {abc }}$ Béatrice Burdin, ${ }^{f}$ \\ Sabine Favre-Bonté, ${ }^{9}$ Rostyslav Bilyy, ${ }^{\text {h }}$ Nicolas Barnich, ${ }^{\text {de }}$ Etienne Fleury, ${ }^{\text {abc }}$ \\ François Ganachaud $^{\mathrm{abc}}$ and Julien Bernard*abc
}

\begin{abstract}
Nanoprecipitation of miglyol into droplets surrounded by a functional glycopolymer generates nanocapsules of biointerest. Fluorophores are trapped in situ or post-grafted onto the crosslinked polymer shell for efficient imaging. The resulting colloids induce aggregation of bacteria through strong specific interactions and promote their facile removal.
\end{abstract}

Sugar moieties play a prominent role in a myriad of biological processes, which explains the latest development of glycomimetics to simulate the attributes of naturally occurring oligo- or polysaccharides. ${ }^{1}$ Monosaccharides generally weakly interact with their receptors, whereas several epitopes grafted on a common scaffold (multivalent molecules, dendrimers, polymer chains) show enhanced activities - thanks to the cluster glycoside effect - for targeting specific glycan-binding proteins. ${ }^{2}$ Owing to their dimensions and multivalent ligand presentation, so-called glyconanomaterials constitute a remarkable platform for applications in imaging, biosensors, targeted drug delivery, enzyme inhibition and so on. ${ }^{3}$ These include metal particles, fullerenes, nanodiamonds, (carbon) nanotubes or polymer nano-assemblies such as micelles, particles or capsules decorated with carbohydrates. ${ }^{3}$

In spite of their significance in biological and medical fields, glyconanocapsules remain far less developed, surely because of their tricky preparation. Most examples of glyconanocapsule

\footnotetext{
${ }^{a}$ Université de Lyon, Lyon, F-69003, France

${ }^{b}$ INSA-Lyon, IMP, Villeurbanne, F-69621, France. E-mail: julien.bernard@insa-lyon.fr

${ }^{c}$ CNRS, UMR 5223, Ingénierie des Matériaux Polymères, Villeurbanne, F-69621, France

${ }^{d}$ Clermont Université, UMR 1071, Inserm/Université d'Auvergne,

63000 Clermont-Ferrand, France

${ }^{e}$ INRA, Unité Sous Contrat 2018, 63000, Clermont-Ferrand, France

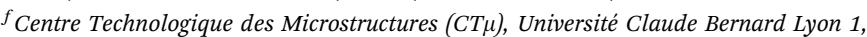
France

${ }^{g}$ Université de Lyon, France Research Group on "Bacterial Opportunistic Pathogens and Environment", UMR 5557 Ecologie Microbienne, CNRS, Vetagro Sup and Université Lyon1, France

${ }^{h}$ Friedrich-Alexander University of Erlangen-Nürnberg, Department of Internal Medicine 3-Rheumatology and Immunology, D-91054 Erlangen, Germany

$\dagger$ Electronic supplementary information (ESI) available. See DOI: 10.1039/c5cc04653j
}

syntheses reported in the literature rely on molecular building blocks or block copolymers self-assembly, ${ }^{4}$ layer-by-layer deposition of glycopolymers on a sacrificial template, ${ }^{5}$ or emulsion approaches. ${ }^{6}$ Recently, we demonstrated that a careful establishment and reading of both oil and polymer phase diagrams allows for setting conditions ensuring the rapid preparation of nanocapsules in a simple batch mixing.7 This concomitant nanoprecipitation/polymer crosslinking procedure (called "Shift'N'Go process") also permits simultaneous functionalization of the shell and filling of the core with molecules of biological interest.

In this communication, we substantiate this proof of concept by preparing biocompatible functional capsules of utility for bio-applications. Particularly, we report on the one-pot synthesis of precisely defined, miglyol-filled, epoxide-functionalized glyconanocapsules and on subsequent post-modifications of the polymer shell via ring opening reactions. We show that the nanocapsules can be easily loaded with actives and that the presence of numerous pendent epoxides within the walls permits an efficient incorporation of relevant molecules such as ligands, probes or metal nanoparticles. Furthermore, the multiple $n$-heptyl $\alpha$-D-mannose motifs present at the capsule surface strongly interact with the lectin sugarbinding sites (adhesin FimH) of adhesive proteinaceous hair-like organelles (type 1 fimbrae) expressed by Escherichia coli (E. coli) to promote adhesion and infection of tissues. ${ }^{8}$ The construction of tag-labelled nanocapsules, with bright fluorophores and/or coated magnetic nanoparticles, is exploited here to monitor aggregation kinetics between the mannosylated nano-objects and AdherentInvasive $E$. coli bacteria (AIEC) and promote bacterial removal.

A water-soluble random copolymer of $N$-[7-( $\alpha$-D-mannopyranosyloxy)heptyl] methacrylamide (HMM) and glycidyl methacrylate (GMA) was prepared by 4-cyano-4-(phenylcarbonothioylthio) pentanoic acid-mediated RAFT polymerization ( $\mathrm{P}\left(\mathrm{HMM}_{206}\right.$-stat-GMA $\left.{ }_{17}\right)$, $M_{\mathrm{nNMR}}=77.1 \mathrm{~kg} \mathrm{~mol}^{-1}, D=1.10$, details in ESI $\dagger$ ). In order to account for the disparity between the relative reactivity ratios of the two monomers $\left(r_{\mathrm{GMA}}=2.82 / 2.77\right.$ and $r_{\mathrm{HMM}}=0.14 / 0.13$ using Jaacks and Kelen-Tüdös methods, respectively, see ESI $\dagger$ ) and the hydrophobicity of GMA, polymerizations were carried out in a semi-batch process using a low molar fraction of GMA (see Experimental part 


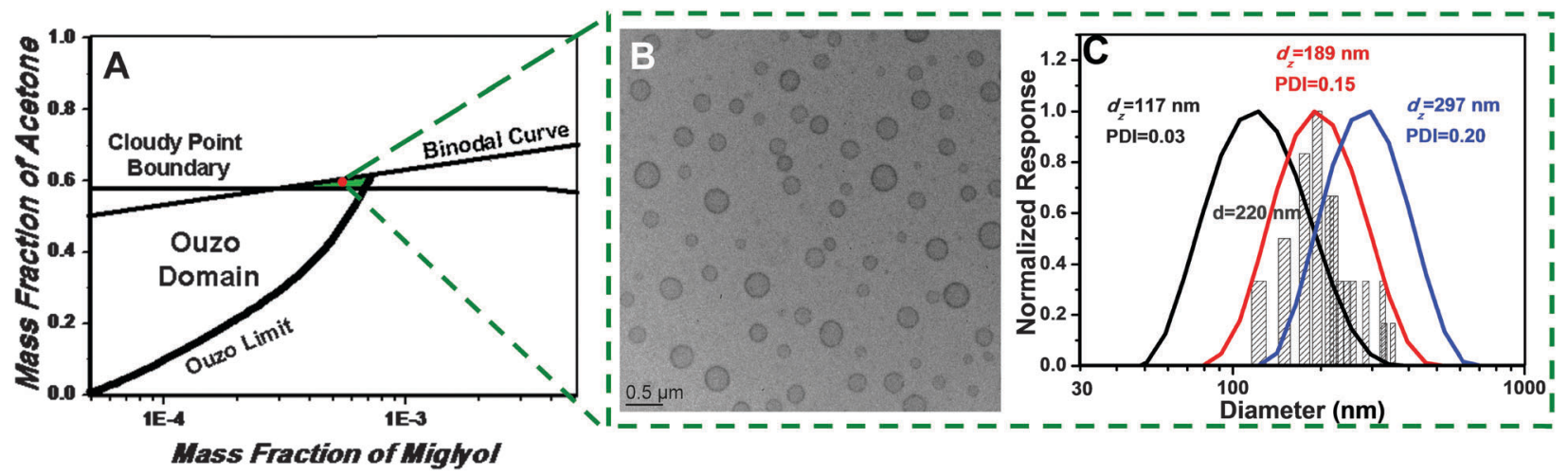

Fig. 1 (A) Overlapped phase diagrams of $\mathrm{P}\left(\mathrm{HMM}_{206}\right.$-stat- $\left.\mathrm{GMA}_{17}\right)$ and miglyol in acetone/water mixtures. The domain in which nanocapsules can be generated through co-nanoprecipitation of miglyol and glycopolymer corresponds to the green zone of (A). Accordingly, nanoprecipitations were performed in the following conditions: polymer content $=0.025 \mathrm{wt} \%$, miglyol content $=0.05 \mathrm{wt} \%$, acetone content $=59 \mathrm{wt} \%$. (B) TEM picture of the resulting glyconanocapsules (C) Comparison of DLS (from left to right, analyses performed in the conditions given in (A), Dulbecco's PBS buffer and distilled water) and TEM size distribution (histogram).
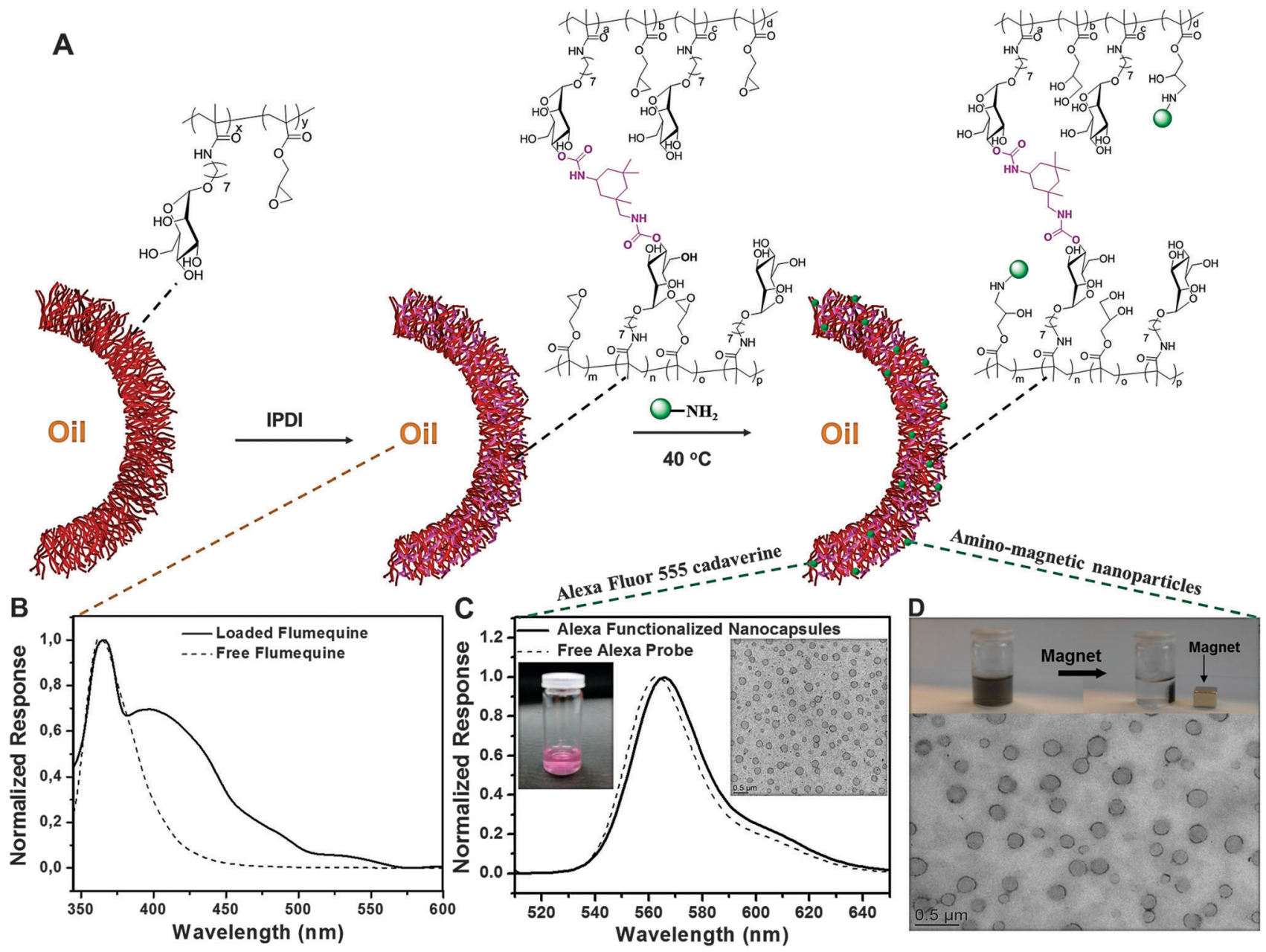

Fig. 2 (A) Route to functional oil-filled glyconanocapsules through epoxy-amine post-nanoprecipitation modifications. (B) Fluorescence emission spectra of an aqueous solution of flumequine and of flumequine-loaded glyconanocapsules dispersion. (C) Fluorescence emission spectrum of AFCfunctionalized glyconanocapsules; inset (top right corner): TEM picture of AFC-functionalized glyconanocapsules (scale bar: $0.5 \mu \mathrm{m}$ ), (top left corner) picture of AFC-functionalized glyconanocapsules. (D) TEM picture of CoNP-grafted glyconanocapsules (bottom) and their magnetically-driven separation from the aqueous solution (top). 
in $\mathrm{ESI} \dagger$ for polymerization details). ${ }^{9}$ This maintains an homogeneous distribution of epoxide groups along the glycopolymer chains and thus avoids the formation of blocky, amphiphilic polymer chains prone to aggregate in water.

Glycopolymer and miglyol phase diagrams were subsequently established in water/acetone mixtures as described in our previous work (see ESI $\dagger$ ). ${ }^{7}$ Polymer/oil/water/acetone compositions affording the simple one-pot preparation of glyconanocapsules via nanoprecipitation are easily identified by overlapping the two diagrams. Typically here, miglyol-filled glyconanocapsules are obtained for mass fractions of acetone and miglyol adjusted to 58-62 wt $\%$ and $0.05 \mathrm{wt} \%$, respectively (see Fig. 1A, green zone). This way, nanocapsules are repeatedly obtained in a simple batch mixing and in the full volume, provided that the solvent shifting procedure is carried out in the correct domain of composition. ${ }^{10}$

Besides, to circumvent potential destruction of the resulting nano-objects in water due to desorption of hydrophilic glycopolymer chains when removing the solvent, the walls of the capsules must be cross-linked (see Fig. $2 \mathrm{~A}$ and ESI $\dagger$ ). Isophorone diisocyanate (IPDI), a low water-sensitive difunctional linker, was used to "freeze" the structure of the nanocapsules through isocyanate/alcohol reactions. Robust spherical nanocapsules were fabricated in a reproducible manner using 48 eq. of IPDI per chain (incorporated in the initial organic solution). Miglyol was proven to remain sequestrated in the inner part of the nanocapsules (see Fig. 1B). TEM pictures confirm the formation of nanocapsules with a diameter of around $220 \mathrm{~nm}$ in the dry state. Depending on the nature of the dispersion medium (nanoprecipitation conditions, PBS buffer or distilled water), particle size and particle size distribution observed by DLS significantly differ ( $z$-average diameter ranging from $117 \mathrm{~nm}$ in acetone/water mixture to $297 \mathrm{~nm}$ in distilled water and PDI from 0.02 to 0.20 , see Fig. 1C) reflecting the inclination of the nanocapsules membranes to substantially swell in favorable conditions. Importantly the glyconanocapsules exhibit good colloidal stability under such conditions and no aggregation is observed.

These $n$-heptyl $\alpha$-D-mannose-functionalized nanocapsules (HM-GC) are intended for bioapplications. In this view, the capsules are loaded with bioactive components in a one-pot procedure (see Fig. 2, Fig. S16 and S17, ESI $\dagger$ ). For instance, a fluoroquinolone antibiotic agent, Flumequine, was encapsulated in glyconanocapsules through dissolution in acetone prior to the nanoprecipitation (Fig. 2B and Fig. S17, ESI $\dagger$ ). Pyrene was also successfully encapsulated using this methodology (Fig. S16, ESI $\dagger$ ). All loadings were assessed by measuring fluorescence emission spectra of the dispersions after dialysis.

The pendent epoxides of the nanocapsules can be used as handles for post-nanoprecipitation attachment of molecules of interest (see Fig. 2A). Owing to the broad applications of the biotin/streptavidin system in biotechnology, we biotinylated the membrane of the capsules with biotin ethylenediamine ( 1 eq. per chain, $12 \mathrm{~h}$ at $40{ }^{\circ} \mathrm{C}$, water). The decoration of the membrane has barely any impact on the dimensions of the nano-objects $\left(D_{z}=111 \mathrm{~nm}, \mathrm{PDI}=0.3\right.$, Fig. S18, ESI $\left.\dagger\right)$; still, the presence of biotin motifs within the shell of the capsules was confirmed by the formation of large aggregates $(\sim 600-700 \mathrm{~nm})$ upon addition of tetrameric avidin and the capability of the glyconanocapsules to disrupt pre-established 4'-hydroxy-azobenzene 2-carboxylic acid/avidin complexes.
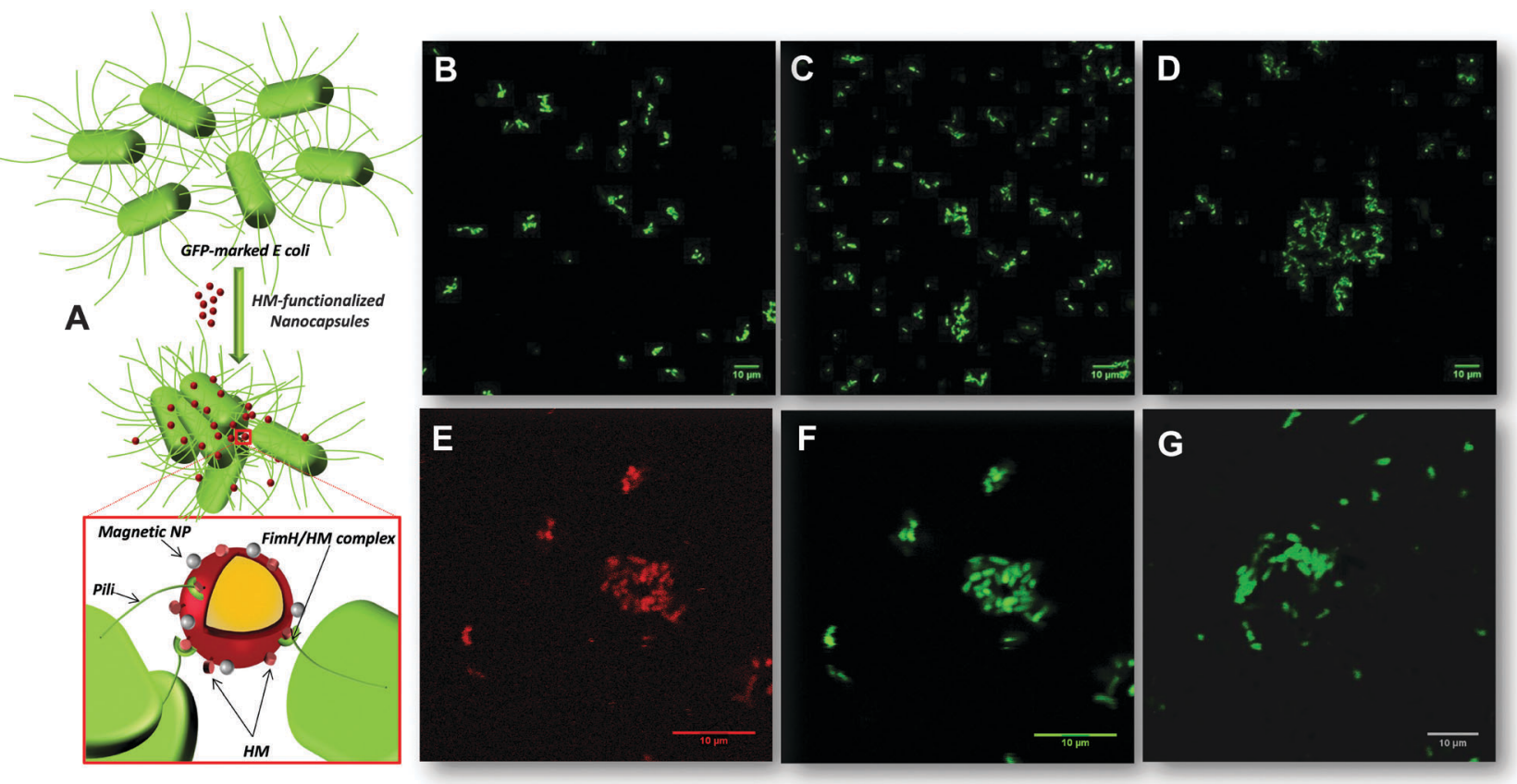

Fig. 3 Left: (A) Principle of bacterial agglutination in the presence of glyconanocapsules. Right: (up): Confocal fluorescence microscopy (CFM) pictures of GFP-marked AIEC LF82 incubated with HM-GC (66 $\mu \mathrm{M})$ for 30 min (B), $2 \mathrm{~h}$ (C) and $3 \mathrm{~h}$ (D). Bottom: (E and F) CFM pictures of GFP-marked AlEC LF82 incubated for $2 \mathrm{~h}$ with AFC-functionalized capsules $(66 \mu \mathrm{M})\left(\lambda_{\text {exc }}=488 \mathrm{~nm}\right.$ for GFP and $543 \mathrm{~nm}$ for AFC). (G) CFM picture of GFP-marked AIEC LF82 clusters extracted from the solution by magnetically-driven separation. 
The glycopolymer membrane can also be functionalized with e.g. fluorescent tags and/or metal nanoparticles. To prove so, Alexa Fluor 555 Cadaverine (AFC) was added to a dispersion of nanocapsules and reacted overnight (1 eq. of AFC per chain, $40{ }^{\circ} \mathrm{C}$ ). After functionalization, AFC-labelled HM-GC having a $z$-average diameter of $123 \mathrm{~nm}$ (PDI $=0.27$ ) and displaying a typical fluorescence emission at $566 \mathrm{~nm}$ were obtained (Fig. 2C and Fig. S19, ESI $\dagger$ ). Likewise, magnetic nanocapsules $\left(D_{z}=126 \mathrm{~nm}\right.$, Fig. 2D and Fig. S20, ESI $\left.\dagger\right)$ were prepared through post-nanoprecipitation modification with amino-functionalized carbon-coated cobalt magnetic nanoparticles (Co-NP, $d \approx 30 \mathrm{~nm}$ ). Thanks to the covalent anchorage of Co-NP on their membranes, glyconanocapsules were rapidly and effectively recovered from the aqueous solution in the presence of a simple magnet (Fig. 2D). Finally, we present below examples of biological assays made possible by these newly developed glyconanocapsules. First, we checked that after purification via dialysis, the epoxyfunctionalized glyconanocapsules are non-toxic (see tests in Fig. S21, ESI $\dagger$ ). Given the remarkable affinity of $n$-heptyl $\alpha$-D-mannose for FimH adhesin (binding affinity of $5 \mathrm{nM}$ recorded by SPR) exposed at the extremity of AIEC type 1 fimbriae, ${ }^{8}$ the capability of HM-GC to interact with AIEC LF82 strains was then explored. In the absence of HM-GC, no clustering of green fluorescent AIEC LF82 $\left(\mathrm{OD}_{620 \mathrm{~nm}}=0.6\right.$, fluorescence emission at $\sim 520 \mathrm{~nm}$ ) is observed after $3 \mathrm{~h}$ suggesting that bacteria alone exhibit a poor tendency to self-aggregate under the conditions of analysis (see ESI $\dagger$ ). Meanwhile, clusters of bacteria are observed when bacteria are incubated with glyconanocapsules in aqueous solution (Fig. 3B and D). The glyconanocapsules efficiently agglutinate $E$. coli thanks to the strong interaction of pendent heptyl mannosides ligated to the membrane and FimH adhesins expressed at the surface of bacteria. Interestingly, the size of bacteria clusters increases with time of incubation (up to $40 \mu \mathrm{m}$ after $3 \mathrm{~h}$ ). The propensity of HM-GC to strongly interact with AIEC bacteria was further corroborated by bacterial adhesion assays. Infection of T84 cells with AIEC $E$. coli. previously incubated with HM-GC at $6 \mu \mathrm{g} \mathrm{mL}^{-1}$ of HM-GC resulted in extremely low level of bacterial adhesion to T84 cells ( $\sim 5 \%$ of residual adhesion, $100 \%$ corresponding to adhesion in absence of any compound see $\mathrm{ESI} \dagger)$.

To assess the specificity of the binding between HM-GC and AIEC LF82, the bacteria were also exposed to nanocapsules built from D-glucose containing glycopolymers (experimental details and TEM pictures given in ESI $\dagger$ ). No cluster of bacteria was observed under such conditions.

HM-GC decorated with AFC allow for visualizing their interactions with $E$. coli. Red emitting glyconanocapsules gradually accumulate at the surface of the bacteria with time to promote interconnections between bacteria and ultimately agglutination. Both AFC and GFP tags produce images of sufficient quality to be overlapped (Fig. 3E and F and Fig. S22, ESI $\dagger$ ). AIEC LF82 strains $\left(\mathrm{OD}_{620 \mathrm{~nm}}=0.6\right)$ were finally incubated at room temperature for $3 \mathrm{~h}$ (after $3 \mathrm{~h}$, [AIEC LF82] $=2 \times 10^{8} \mathrm{~mL}^{-1}$ ) with magneto-responsive HM-GC $(0.5 \mathrm{mg}$ in $100 \mu \mathrm{L})$ and a magnet was placed close to the suspension to promote the separation of the magneto-responsive materials from the solution (Fig. 2D). ${ }^{11}$ Under such conditions, the nanocapsules achieved high yields of bacteria removal (83\%, see details in ESI $\dagger$ ). Consistent with these results, confocal fluorescence microscopy characterization of the (extracted and re-dispersed) magneto-responsive materials confirmed the formation of stable HM-GC/bacteria clusters (Fig. 3G).

In conclusion, we have demonstrated that oil-filled, shellfunctionalized glyconanocapsules can be reproducibly built and loaded with actives in a simple one-pot procedure. Epoxide-functionalized nanocapsules constitute a convenient platform for post-attachment of biologically relevant molecules or CoNP through epoxide-amine ring opening reactions. Thanks to the concomitant presence of numerous mannose residues and fluorophores or CoNP, kinetics of $E$. coli agglutination in the presence of the nanocapsules can be conveniently monitored and the resulting bacterial clusters are efficiently extracted from solution via magnetically-driven separation.

X.Y acknowledges the CSC for a PhD grant. This work was carried out with financial support from the French Agence Nationale de la Recherche (STARLET, ANR-12-BSV5-0016-01). The authors thank Dr Stéphane Chambert and Dr Xuejiao Jiang for fruitful discussions and bacteria counting.

\section{Notes and references}

1 R. A. Dwek, Chem. Rev., 1996, 96, 683.

2 J. J. Lundquist and E. J. Toone, Chem. Rev., 2002, 102, 555.

3 (a) N. C. Reichardt, M. Martin-Lomas and S. Penades, Chem. Soc. Rev., 2013, 42, 4358; (b) R. Sunasee and R. Narain, Macromol. Biosci., 2013, 13, 9; (c) X. Li and G. Chen, Polym. Chem., 2015, 6, 1417; (d) L. Zhao, Y. Chen, J. Yuan, M. Chen, H. Zhang and X. Li, ACS Appl. Mater. Interfaces, 2015, 7, 5177; (e) M. Khanal, F. Larsonneur, V. Raks, A. Barras, J.-S. Baumann, F. A. Martin, R. Boukherroub, J.-M. Ghigo, C. Ortiz Mellet, V. Zaitsev, J. M. Garcia Fernandez, C. Beloin, A. Siriwardena and S. Szunerits, Nanoscale, 2015, 7, 2325.

4 (a) J. Huang, C. Bonduelle, J. Thevenot, S. Lecommandoux and A. Heise, J. Am. Chem. Soc., 2012, 134, 119; (b) J. Voskuhl, M. C. A. Stuart and B. J. Ravoo, Chem. - Eur. J., 2010, 16, 2790; (c) L. Gallego-Yerga, M. Lomazzi, F. Sansone, C. Ortiz Mellet, A. Casnati and J. M. Garcia Fernandez, Chem. Commun., 2014, 50, 7440 .

5 H. L. Guo, Q. Q. Guo, T. C. Chu, X. G. Zhang, Z. M. Wu and D. M. Yu, J. Mater. Sci.: Mater. Med., 2014, 25, 121.

6 (a) R. Roux, L. Sallet, P. Alcouffe, S. Chambert, N. Sintes-Zydowicz, E. Fleury and J. Bernard, ACS Macro Lett., 2012, 1, 1074; (b) K. Malzahn, F. Marsico, K. Koynov, K. Landfester, C. K. Weiss and F. R. Wurm, ACS Macro Lett., 2014, 3, 40; (c) R. H. Utama, Y. Jiang, P. B. Zetterlund and M. H. Stenzel, Biomacromolecules, 2015, 16, 2144.

7 X. Yan, M. Delgado, A. Fu, P. Alcouffe, S. G. Gouin, E. Fleury, J. L. Katz, F. Ganachaud and J. Bernard, Angew. Chem., Int. Ed., 2014, 53, 6910.

8 X. Yan, A. Sivignon, N. Yamakawa, A. Crepet, C. Travelet, R. Borsali, T. Dumych, Z. Li, R. Bilyy, D. Deniaud, E. Fleury, N. Barnich, A. Darfeuille-Michaud, S. G. Gouin, J. Bouckaert and J. Bernard, Biomacromolecules, 2015, 16, 1827.

9 P. Escale, S. R. S. Ting, A. Khoukh, L. Rubatat, M. Save, M. H. Stenzel and L. Billon, Macromolecules, 2011, 44, 5911.

10 Choosing HD as oil, the domain for the formation of nanocapsules is much larger. Then, the final content of acetone ranges from 58 to $70 \mathrm{wt} \%$ and the final weight fraction of $\mathrm{HD} \sim 0.05 \mathrm{wt} \%$ (Fig. S14 and $\mathrm{S} 15, \mathrm{ESI} \dagger)$.

11 (a) D. M. Hatch, A. A. Weiss, R. R. Kale and S. S. Iyer, ChemBioChem, 2008, 9, 2433; (b) M. Bera, N. Azzouz, S. Schmidt, D. V. Volodkin, S. Mosca, M. Chanana, P. H. Seeberger and L. Hartmann, Biomacromolecules, 2013, 14, 1927. 